\title{
Influência de escolares participantes de um programa de educação nas práticas diárias de saúde bucal em seu ambiente familiar
}

\section{Influence of schoolchildren participating in an oral health education program on their family's practices}

\author{
Gabriela Nascimento Salles ${ }^{1}$, Marina Berti ${ }^{2}$, Daniela Pereira Lima ${ }^{2}$, \\ Mariângela Monteiro de Melo Baltazar ${ }^{3}$, Brenda Rex Machado ${ }^{4}$, \\ Helena Pfeffer ${ }^{5}$, André Luiz Marçal Terreri ${ }^{6}$
}

\begin{abstract}
Resumo
Este estudo objetivou verificar a influência de escolares participantes de um programa de educação em saúde bucal nas práticas diárias de sua família, por meio da percepção de seus pais e/ou responsáveis legais. A pesquisa, caracterizada como um estudo exploratório descritivo com abordagem quanti-qualitativa, foi composta por 146 pais e/ou responsáveis de escolares com idade entre 4-12 anos. Os dados foram coletados por meio de questionário estruturado, autoaplicado, com perguntas abertas e fechadas. As questões propostas abordaram conhecimento por parte dos pais sobre as atividades de educação em saúde bucal realizadas na escola, a importância que os mesmos atribuem a essas atividades, bem como o aprendizado com os filhos e a existência de mudança de hábitos em casa. Ao todo, $86 \%$ dos entrevistados tinham conhecimento das atividades educativas e preventivas desenvolvidas na escola e todos relataram ser importante tais atividades, principalmente no que se refere à saúde, cuidado e orientação das crianças. A maioria dos participantes da pesquisa (75\%) relatou ter aprendido algo referente à saúde bucal com seus filhos, e desses, 30\% citaram a escovação como sendo o fator de maior aprendizado. Além disso, $76 \%$ relataram a existência de mudanças nos hábitos de saúde bucal de sua família. Pode-se concluir que os escolares foram capazes de transmitir o conhecimento adquirido na escola para seus familiares, o que pode contribuir para a mudança da rotina de saúde bucal da sua família.
\end{abstract}

Palavras-chave: Educação em saúde bucal; Promoção da saúde; Serviços de saúde escolar.

${ }^{1}$ Mestranda em Odontologia na Universidade Estadual do Oeste do Paraná (Unioeste), Cascavel, Paraná, Brasil. E-mail: gabriela. nascsalles@hotmail.com

${ }^{2}$ Doutorado em Odontologia Preventiva e Social pela Faculdade de Odontologia de Araçatuba da Universidade Estadual Paulista (FOA-Unesp), Araçatuba, São Paulo, Brasil. Professora da Universidade Estadual do Oeste do Paraná, Cascavel, Paraná, Brasil.

${ }^{3}$ Doutorado em Odontologia pela Pontifícia Universidade Católica do Paraná (PUCPR), Curitiba, Paraná, Brasil. Professora da Universidade Estadual do Oeste do Paraná, Cascavel, Paraná, Brasil.

${ }^{4}$ Graduanda em Odontologia na Universidade Estadual do Oeste do Paraná, Cascavel, Paraná, Brasil.

${ }^{5}$ Mestranda em Odontologia na Universidade Estadual do Oeste do Paraná, Cascavel, Paraná, Brasil.

${ }^{6}$ Doutorado em Ciências da Saúde pela Faculdade de Medicina de São José do Rio Preto (Famerp), São José do Rio Preto, São Paulo, Brasil. Professora Adjunta da Universidade Estadual do Oeste do Paraná, Cascavel, Paraná, Brasil. 


\begin{abstract}
This study aimed to verify the influence of schoolchildren participating in an oral health education program on their family's practices, through the perception of their parents and / or legal guardians. A research, characterized as a descriptive exploratory study, with quantitative and qualitative approach was composed of 146 parents and / or guardians of students aged 4-12 years. Data were collected through a structured questionnaire, applied automatically, with open and closed questions. The questions approached address the knowledge of parents about oral health education activities performed at school, the importance they attach to these activities, as well as learning with their children and changing habits at home. In performing $86 \%$ of respondents were aware of educational and preventive activities developed at school and all reported to be important activities, especially with regard to health, care and guidance of children. Most survey participants (75\%) reported having learned something related to oral health from their children and $30 \%$ cited brushing as the factor of greatest learning. In addition, $76 \%$ reported changes in their family's oral health habits. It can be concluded that the students were able to transmit the knowledge acquired at school to their family members, which can contribute to changing your family's oral health routine.
\end{abstract}

Keywords: Dentalhealth education; Health promotion; School health services.

\section{Introdução}

Ações educativas e preventivas consistem em excelente meio de proteção a doenças bucais. Entretanto, ainda há um limitado conhecimento por parte de algumas populações, principalmente as de baixo nível socioeconômico, no que tange à prevenção dessas doenças. ${ }^{(1-2)}$

Desta forma, para promover saúde, a educação constitui um dos pontos fundamentais, especialmente porque visa a busca pela capacitação e pelo encorajamento do ser humano a assumir responsabilidades sobre a sua própria saúde e sua participação na vida comunitária. Educar nesse âmbito é estimular o desenvolvimento de habilidades, a formação de atitudes e a criação de valores que levem o indivíduo a agir no seu dia a dia em benefício da própria saúde bucal e da saúde bucal dos outros. ${ }^{(3)}$

A educação em saúde bucal para crianças é considerada prioritária, devido ao alto risco à cárie dentária nessa idade, ${ }^{(4)}$ assim como a suas constantes mudanças no ambiente bucal, a facilidade de mudar hábitos errôneos e maior facilidade de aprendizagem.
$\mathrm{O}$ atendimento escolar às crianças de $0 \mathrm{a}$ 3 anos e de 4 a 5 anos é, fundamentalmente, um desafio dos municípios no Brasil. As redes municipais respondem por $71,6 \%$ das matrículas de educação infantil e, junto com as escolas privadas, receberam um total de 8,7 milhões de crianças em todo o País em 2018. ${ }^{(5)}$ Atualmente, 128,4 mil escolas brasileiras recebem 15,2 milhões de alunos no ensino fundamental - anos iniciais -, e 12 milhões de alunos no ensino fundamental anos finais -, um grande contingente populacional que dá a dimensão dos desafios a serem enfrentados. A rede pública é a responsável por atender a grande maioria dos 27,2 milhões de alunos dessa etapa. No território nacional, a cada cinco alunos, um está na rede privada e quatro estão na rede pública. ${ }^{(5)} \mathrm{O}$ setor educacional, dada sua capilaridade e abrangência, é um aliado importante para a concretização de ações de promoção da saúde. Ações voltadas para o fortalecimento das capacidades das crianças e adolescentes, para tomada de decisões favoráveis à comunidade, para a criação de ambientes saudáveis e para a consolidação de uma política intersetorial, ampliam a qualidade de vida das comunidades. ${ }^{(6)}$ 
A importância dos programas de educação em saúde bucal nas escolas é relatada por determinados autores, principalmente no que se refere ao aprendizado e comportamento positivo das crianças. ${ }^{(7-8)}$ Muitas pesquisas enfocam os programas de educação em saúde bucal, evidenciando a relação entre a condição de saúde bucal e o conhecimento e atitudes em saúde bucal de escolares..$^{(9-13)}$

Contudo, é pouco abordado na literatura o fato de que a criança tem condições de aplicar em seu cotidiano a experiência vivenciada na escola e pode agir como agente multiplicador de saúde dentro do ambiente familiar. ${ }^{(14)}$

Desta maneira, o presente estudo tem como objetivo verificar a influência de escolares participantes das atividades educativas e preventivas contempladas em um programa de educação em saúde bucal nas práticas diárias de saúde bucal de sua família.

\section{Material e Método}

A pesquisa, caracterizada como um estudo exploratório descritivo com abordagem quantiqualitativa, foi composta por pais e/ou responsáveis de escolares de 4 a 12 anos de idade, participantes do projeto de extensão denominado "Tratamento Restaurador Atráumatico no Controle da Cárie em Escolares do Cascavel Velho - PR", matriculadas no período vespertino da Escola Municipal Irene Rickli, pertencente ao bairro Cascavel Velho, do município de Cascavel, estado do Paraná, Brasil, no ano de 2017. O bairro Cascavel Velho fica localizado na Região Sul do município de Cascavel e pertence a uma área de alta vulnerabilidade social do município.

O referido projeto de extensão é desenvolvido por estudantes e professores do Curso de Odontologia da Universidade Estadual do Oeste do Paraná (Unioeste), Cascavel, Paraná, Brasil, e tem como objetivo proporcionar aos escolares o desenvolvimento de suas capacidades cognitivas, afetivas e psicomotoras, relativas à saúde bucal (cadastro institucional 50243/2017).
Para definir a amostra, levou-se em consideração o número de todos os escolares, com idade entre 4 e 12 anos, participantes de um projeto de educação em saúde bucal, totalizando 171 crianças e, consequentemente, o mesmo número de pais e/ ou responsáveis. Entretanto, 25 (14,6\%) dos pais e/ ou responsáveis foram excluídos da amostra, pois não concordaram em participar da pesquisa ou não devolveram o instrumento de coleta de dados preenchido, totalizando uma amostra final de 146 participantes.

No estudo, os dados foram coletados através de questionário adaptado de Garbin et al., ${ }^{(15)}$ estruturado, autoaplicado, com seis perguntas abertas e dez perguntas fechadas. Estes questionários foram enviados pela agenda dos alunos, que consiste no mecanismo adotado de comunicação entre a escola e as famílias. As questões propostas abordaram conhecimento por parte dos pais sobre as atividades de educação em saúde bucal realizadas na escola, a importância que os pais atribuem a essas atividades, aprendizado com os filhos e a existência de mudanças de hábitos em casa.

Para análise quantitativa dos dados foi utilizado o software XLSTAT 2016, ${ }^{(16)}$ por meio da distribuição das frequências e porcentagens. Para a análise qualitativa, utilizou-se a análise de conteúdo proposta por Bardin, por meio da técnica de análise de categorias. ${ }^{(17)}$

O estudo recebeu aprovação do Comitê de Ética em Pesquisa da Universidade Estadual do Oeste do Paraná (CAAE: 78320117.0.0000.0107).

\section{Resultados}

A amostra em estudo foi composta por 146 indivíduos. Em relação às atividades educativas e preventivas em saúde bucal contempladas pelo projeto de extensão (distribuição de kits de higiene bucal, orientações em saúde bucal, escovação supervisionada e palestras educativas) realizadas na escola (Q1), a maioria dos pais afirmou ter conhecimento sobre tais atividades (86\%), conforme pode se verificar na Tabela 1. 
Tabela 1 - Frequências absolutas (FA), relativas (FR \%) e total de indivíduos. p: valor do teste do quiquadrado para aderência e k proporções. $(n=146) .{ }^{*}$ Nenhuma alternativa. Cascavel, PR, 2017.

\begin{tabular}{llccc}
\hline Questão & Categorias & FA & FR \% & p \\
\hline \multirow{2}{*}{ Q1 - Atividades educativas e preventivas? } & Não & 20 & 14 & $<0,05$ \\
& Sim & 126 & 86 & \\
\hline \multirow{2}{*}{ Q2 - Essas atividades são importantes? } & Sim & 146 & 100 & \\
\hline & Saúde & 61 & 21 \\
& Cuidado & 40 & 14 \\
& Orientação & 32 & 11 & \\
& Necessidade & 29 & 10 & \\
& Higiene & 25 & 9 & \\
& Prevenção & 20 & 7 & \\
& Escovação & 19 & 7 & \\
& Conhecimento & 15 & 5 & \\
& Bom & 13 & 4 & \\
& Incentivo & 8 & 3 & \\
& Outros & 19 & 7 & \\
& NA* & 11 & 4 & \\
\hline
\end{tabular}

Fonte: os autores.

Questionados sobre a importância dessas atividades (Q2), todos os entrevistados concordaram com a necessidade de haver medidas preventivas e educativas em relação à saúde bucal. Com isso, a maioria deles justificou a importância de tais práticas $(\mathrm{Q} 3)$ devido a fatores relacionados à saúde $(21 \%)$, cuidados $(14 \%)$, orientações $(11 \%)$ e necessidades $(10 \%)$, sendo essas categorias os pares de proporções significativamente diferentes dos outros (Tabela 1 e Figura 1).

Figura 1 - Nuvem de palavras - importância para os pais das atividades de educação e prevenção em saúde bucal realizadas na escola. Cascavel, PR, 2017.

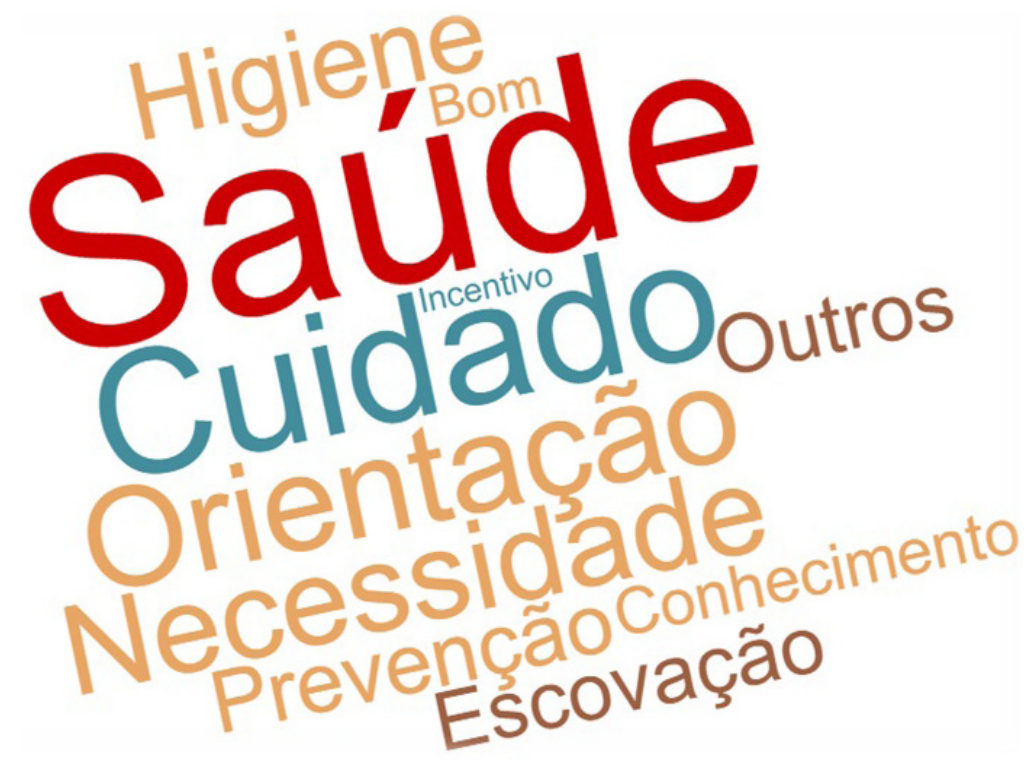

Fonte: os autores. 
Quando questionados se aprenderam algo quando interrogados sobre o que aprenderam (Q5), sobre saúde bucal com os filhos (Q4), a maioria dos as respostas mais comuns foram "escovação", com pais $(75 \%)$ respondeu positivamente. No entanto, 30\%, e "NA", com 34\% (Tabela 2).

Tabela 2 - Frequências absolutas (FA), relativas (FR \%) e total de indivíduos. p: valor do teste do quiquadrado para aderência e k proporções. $(\mathrm{n}=146)$. *Nenhuma alternativa. Cascavel, PR, 2017.

\begin{tabular}{llccc}
\hline Questão & Categorias & FA & FR \% & p \\
\hline \multirow{2}{*}{ Q4 - Aprendeu algo com seu filho? } & Não & 33 & 23 & \\
& Sim & 110 & 75 & $<0,05$ \\
& NA* & 3 & 2 & \\
& Escovação & 52 & 30 & \\
& Cuidado & 14 & 8 & \\
& Saúde bucal & 13 & 8 & \\
Q5 - O quê? & Prevenção & 9 & 5 & \\
& Fio dental & 8 & 5 & \\
& Higiene & 6 & 4 & \\
& Importante & 4 & 2 & \\
& Outros & 7 & 4 & \\
\hline
\end{tabular}

Fonte: os autores.

A maioria dos entrevistados $(76 \%)$ re- principais escolhas foram as categorias: cuidados latou uma mudança na rotina de saúde bucal de (24\%), escovação (17\%) e compromisso (13\%), sua família após as atividades (Q6), sendo obtida sendo essas opções, juntamente com "NA" (34\%), diferença significativa entre as respostas. Quando os pares de proporções significativamente diferenquestionados sobre qual mudança ocorreu (Q7), as tes dos outros (Tabela 3).

Tabela 3 - Frequências absolutas (FA), relativas (FR \%) e total de indivíduos. p: valor do teste do quiquadrado para aderência e k proporções. $(\mathrm{n}=146)$. *Nenhuma alternativa. Cascavel, PR, 2017.

\begin{tabular}{llccc}
\hline Questão & Categorias & FA & FR \% & p \\
\hline \multirow{2}{*}{ Q6 - Houve alguma mudança nos hábitos? } & Não & 30 & 21 & \\
& Sim & 111 & 76 & $<0,05$ \\
& NA* & 5 & 3 & \\
\hline & Cuidado & 46 & 24 & \\
& Escovação & 33 & 17 & \\
& Compromisso & 25 & 13 & \\
& Saúde & 11 & 6 & \\
Q7 - O que mudou? & Exemplo & 7 & 4 & \\
& Prevenção & 7 & 4 & $<0,05$ \\
& Subjetivos & 7 & 4 &
\end{tabular}




\begin{tabular}{llccll} 
& Fio dental & 3 & 2 & \\
& Dieta & 1 & 1 & \\
& NA* & 48 & 25 & \\
\hline & Péssima & 4 & 3 & \\
Q8 - Como considera a sua saúde bucal? & Ruim & 9 & 6 & \\
& Regular & 43 & 29 & $<0,05$ \\
& Boa & 74 & 51 & \\
& Ótima & 16 & 11 & \\
\hline & Péssima & 2 & 1 & \\
& Ruim & 14 & 10 & \\
& Regular & 38 & 26 & $<0,05$ \\
& Boa & 70 & 48 & \\
& Ótima & 22 & 15 & \\
\hline
\end{tabular}

Fonte: os autores.

Questionados sobre como consideram sua própria saúde bucal (Q8), a maioria dos entrevistados $(51 \%)$ relatou ter boa sua saúde bucal. Padrão semelhante foi observado nas respostas em relação à saúde dos filhos (Q9), sendo que a maior parte (48\%) a considera boa (Tabela 3$)$.

\section{Discussão}

No Brasil, dados do levantamento de saúde bucal realizado em 2010 revelaram que $27 \%$ das crianças de 18 a 36 meses apresentam, no mínimo, um dente com experiência de cárie, e a proporção chega a $45 \%$ em crianças de 5 anos de idade. ${ }^{(4)}$ Dessa forma, a cárie na infância é considerada um problema de saúde pública, pois atinge um grande número de crianças, gerando impacto negativo na qualidade de vida devido a problemas no desenvolvimento e no bem-estar dessas crianças, pois a cárie pode gerar dor, abscesso e infecção, o que impede a realização de uma alimentação adequada e afeta a autoestima, a sociabilidade e o bem-estar da criança. ${ }^{(18)}$

Deste modo, percebe-se a importância de ações educativas e preventivas para esse grupo, desde cedo, com a finalidade de promover a aquisição de hábitos saudáveis e diminuir os índices de cárie e doenças periodontais, diminuindo, com isso, o risco de adquirir essa doença no futuro. Conforme conceito contido na Carta de Ottawa, o termo "educação" é um dos pontos centrais para a promoção de saúde, prática voltada para a melhoria da saúde das populações. Não somente a educação formal, mas toda ação educativa que propicie a reformulação de hábitos, aceitação de novos valores e que estimule a criatividade. ${ }^{(19)}$

De acordo com a Organização Mundial da Saúde (OMS), ${ }^{(6)}$ as escolas são locais propícios para o desenvolvimento de programas educativos em saúde, pois envolvem toda a sociedade escolar, ou seja, familiares, professores e comunidade. ${ }^{(6)}$ Isso ficou evidenciado nesta pesquisa, onde $76 \%$ dos pais relataram alguma mudança nos hábitos de sua família, evidenciando o envolvimento de toda família no processo educativo.

Programas educativos escolares precisam ter como objetivo a promoção de saúde e a transformação, quando necessária, de crenças, atitudes e comportamentos em relação à saúde. ${ }^{(20-21)} \mathrm{Du}$ rante sua realização deve-se buscar o domínio de habilidades e o despertar da imaginação, estimular a cooperação e compreensão, e saber respeitar, explorar e ampliar os saberes que toda criança possui, especialmente quando chega à escola. ${ }^{(22)}$ 
A importância dos programas de educação em saúde nas escolas de educação infantil tem respaldo científico, tanto na aquisição de novos conhecimentos, como na redução dos índices das doenças bucais. ${ }^{(23-27)}$ Neste estudo pode ser verificada a aquisição de conhecimentos em saúde bucal por parte das crianças, por meio do que as mesmas foram capazes de transmitir para os seus pais, visto que $75 \%$ dos mesmos relataram ter aprendido algo referente à saúde bucal com seus filhos.

Menezes, ${ }^{(28)}$ em um estudo que analisou a participação da família na promoção da saúde bucal de alunos contemplados por um programa educativo e preventivo realizado em uma escola pública no Nordeste brasileiro, verificou que 95\% dos pais tinham conhecimento dos procedimentos de saúde bucal desenvolvidos nas escolas, embora não determinassem de maneira precisa sua periodicidade e importância. Além disso, todos os familiares entrevistados demonstraram um elevado grau de satisfação e ressaltaram a importância de participar da atenção odontológica aos escolares. Esses resultados corroboram os achados da presente pesquisa, onde $86 \%$ dos pais tinham conhecimento das atividades desenvolvidas na escola e todos relataram ser importante tais atividades, principalmente no que se refere à saúde, cuidado e orientação das crianças.

Uma das grandes dificuldades encontradas na realização de programas educativos em saúde bucal é a indisposição que os indivíduos têm em adquirir novos hábitos ou modificar seus hábitos em relação aos cuidados com a higiene bucal. ${ }^{(29)}$ Entretanto, foi verificado neste estudo, por meio da percepção dos pais, que as crianças participantes desse programa aumentaram o interesse em cuidar dos dentes, influenciando na mudança de hábitos da família. Além disso, as crianças realmente levam para casa o que aprendem na escola; essa percepção ficou clara nas citações dos pais quando indagados sobre o que aprenderam com seus filhos.

Todavia, para que os objetivos de um programa educativo escolar sejam alcançados, para que realmente a criança aprenda e seja capaz de influenciar o seu ambiente familiar, o ensino deve ser pautado nas necessidades das crianças e deve ser centrado em uma metodologia participativa, problematizadora e, sobretudo, motivadora. A criança deve ser motivada, ou seja, o educador deve despertar o interesse pelo assunto a ser abordado. ${ }^{(15)}$ Destarte, na realização do processo educativo para crianças escolares é essencial que o dentista, no papel de educador, saiba além do conhecimento técnico, atue como agente promotor de saúde, com conhecimento sobre Ciências Sociais e Psicologia, sempre lance mão das professoras como agentes multiplicadores e, de forma ampla, faça o uso de recursos didático-pedagógicos, principalmente de atividades lúdicas.

Vale ressaltar a importância do envolvimento de estudantes de Odontologia em programas de educação em saúde bucal, pois esses programas dão a oportunidade aos mesmos de aprendizagem na área de educação em saúde, realização de pesquisas voltadas à saúde da criança e estreitamento de relações entre a universidade e a comunidade. Essa experiência é extremamente válida, porque fora do ambiente acadêmico tradicional, o aluno tem oportunidade de desenvolver sensibilidade e responsabilidade social, por meio de uma participação mais ativa junto à comunidade. ${ }^{(15,28)}$

\section{Conclusão}

Pode-se constatar, por meio da percepção dos pais, que os pré-escolares foram capazes de transmitir o conhecimento adquirido na escola para seus familiares, o que pode contribuir para a mudança da rotina de saúde bucal da sua família. Assim, percebe-se a importância da realização de programas educativos em saúde bucal para crianças que frequentam as escolas, com a finalidade de promover saúde bucal para toda a família.

\section{Referências}

1 Rajab LD, Petersen PE, Bakaeen G, Hamdan MA. Oral health behaviour of schoolchildren and parents in Jordan. Int. J. Paediatr. Dent. 2002;12: 168-76. 
2 Anselmo TM, Brunson DK, Martinez ME, Christensen M, O'Connell J. Expanding School-Based Sealant Programs to Realize Treatment Cost Savings in Colorado. J Dent Hyg. 2007;4:88.

3 Bijella MFTB, Bijella VT, Figueiredo MC. Avaliação de um programa odontológico, com bases educativas, preventivas e curativa, desenvolvido com pré-escolares durante 12 meses. Cecade News.1995;3(2):1-5.

4 Ministério da Saúde (BR). Projeto SB Brasil 2010. Pesquisa Nacional de Saúde Bucal: resultados principais. Brasília: Ministério da Saúde; 2011.

5 Ministério da Educação (BR). Anuário brasileiro da educação básica 2019. São Paulo: Editora Moderna; 2019.

6 World Health Organization. Ottawa charter for health Promotion. First International Conference of Health Promotion. Ottawa: WHO; 1986.

7 Ohara S, Kawaguchi Y, Shinada K, Sasaki Y. Evaluation of school-based dental health activities including fluoride mouth-rinsing in Hiraizumi, Japan. J Med Dent Sci, 2000;47(2): 133-41.

8 Jenkins SR, Geurink K V. A Rural School-Based Oral Health Program. J Dent Hyg. 2006;1:26.

9 Buischi YP, Axelsson P, Siqueira TRF. Controle mecânico do biofilme dental e a prática de promoção de saúde bucal. In: Buischo, YP. Promoção de saúde bucal odontológica. São Paulo: Artes Médicas; 2000. cap. 8, p. 169-214. (Série EAP - APDC, v. 22).

10 Kinnby CG, Palm L, Widenheim J. Evaluation of information on dental health care at child health centers: differences in educational level, attitudes, and knowledge among parents of preschool children with different caries experience. Acta Odontol Scand. 1991;49(5): 289-95.

11 Peng B, Petersen PE, Tai BJ. Changes in oral health knowledge and behaviour 1987-95 among inhabitants of Wuhan City, PR China. Int Dent J 1997;47:142-7.
12 Petersen PE. Effectiveness of oral health care: Danish experiences. Proc Finn Dent Soc 1992;88(1-2):13-23.

13 Van Palenstein Helderman WH, Benzian H. Implementation of a basic package of oral care: towards a reorientation of dental NGOs and their volunteers. Int Dent J.2006;56:44-6.

14 Kwan SL, Petersen EP, Pine CM, Borutta A. Health-promotingschools: an opportunity for oral health Promotion. Bull World Health Organ. 2005 Sep;83(9):677-85.

15 Garbin C, Garbin A, Santos K, Lima D. Oral health education in schools: promoting health agents. Int J Dent Hyg. 2009 Aug;7(3):212-6.

16 Addinsoft. XLSTAT 18.06 Your Data Analysis Solution. New York: Addinsoft; 2016.

17 Bardin L. Análise de conteúdo. Lisboa: Edições 70; 1994.

18 Losso EM, Tavares MC, Silva JY, Urban CA. Severe early childhood caries: an integral approach. J Pediatr. 2009;85(4):295-300.

19 American Academy of Pediatric Dentistry. Clinical guideline on baby bottle tooth decay/ early childhood caries/breastfeeding/early childhood caries: unique challenges and treatment options.[Internet]. [cited 2018 Nov 15]. Available from: https://bit.ly/3hmYdTe

20 World Health Organization. Research to improve implementation and effectiveness of school healtprogrammes. In: Expert Committee on Comprehensive School Health Education and Promotion. Report of the School Working Group and the WHO. Geneva: World Health Organization; 1996.

21 Baggio MA, Berres R, Gregolin BPS, Aikes S. Implantação do Programa Saúde na Escola em Cascavel, Paraná: relato de enfermeiros. Rev Bras Enferm. 2018;71(suppl 4):1631-8.

22 Ministério da Saúde (BR). A promoção da saúde no contexto escolar. Rev Saúde Pública. 2002;36(2):533-5.

23 Carvalho FFB. A saúde vai à escola: a promoção da saúde em práticas pedagógicas. Physis. 2015;25(4):1207-27. 
24 Casemiro JP, Fonseca ABC, Seco FVM. Promover saúde na escola: reflexões a partir de uma revisão sobre saúde escolar na América Latina. Ciênc. Saúde Colet. 2014;19(2):829-40.

25 Lopes IE, Nogueira JAD, Rocha DG. Eixos de ação do Programa Saúde na Escola e Promoção da Saúde: revisão integrativa. Saúde Debate 2018;42(118):773-89.

26 Gomes JP. As Escolas Promotoras de Saúde: uma via para promover a saúde e a educação para a saúde da comunidade escolar. Educação. 2009;32(1):84-91.

27 Pankai G, Sehgal M, Mittal R. Evaluating the effectiveness of school-based dental health education program among children of different socioeconomic groups. J Indian SocPedodPrev Dent. 2005;23(3):131-3.

28 Menezes AF. A participação da família na Promoção da saúde bucal em alunos da escola Raimundo Pimentel Gomes - CAIC, atendidos pelo PSF - CAIC, em 2002, Sobral - Ceará [Monografia]. Sobral: Universidade Estadual Vale do Acaraú, 2004.

29 Arcieri RM, Garbin CAS, Santo CA, Takano RY, Gonçalves PE. The Influence of the motivation and of the supervised brushing in habits of $\mathrm{L}$ oral hygiene in Brazilian preschooler. Acta Odontol. Venez. [Internet]. 2007[citado 2019 mar 8];5(4). Disponível em: https://bit. ly/3hajEbu 
